\title{
B-10. Dynamics of Lymphocytes in the Peripheral Blood in Brain Tumor Patients
}

Takashi Shimzu, Nagao Kubo and Koichi Krtamura Kintomo TAKakURa

Department of Neurosurgery, Tokyo Women's Medical College Department of Neurosurgery, National Cancer Center Hospital 